\title{
Predicting rehabilitation treatment helpfulness to stroke patients: A supervised learning approach
}

\author{
Chia-Lun Lo*1, Hsiao-Ting Tseng ${ }^{2}$ \\ ${ }^{1}$ Department of Health Business Administration, Fooyin University, Taiwan \\ ${ }^{2}$ Institute of Information Management, National Chiao Tung University, Taiwan
}

Received: December 1, 2016

Accepted: February 16, 2017

Online Published: February 27, 2017

DOI: $10.5430 /$ air.v6n2p1

URL: https://doi.org/10.5430/air.v6n2p1

\begin{abstract}
Stroke (Cerebral vascular accident, CVA) is a common and serious disease. Most of the survivals would be disabled after their illness recovery, causes serious burden on caregivers. It is said that rehabilitation could help functional recovery of stroke patients, regain independence after stroke. Due to the long course of stroke, how to prevent survivals from recurrence is an important issue. This study attempts to examine the relationship between stroke recurrence and strength of rehabilitation, and build a stroke recurrence prediction model utilizing a number of supervised learning techniques to assist physicians with making clinical decisions.

In the past, most of the related work used the samples from a single hospital as a sample, but it cannot fully catch all the clinic information of the patients. Therefore, this study used the Longitudinal Health Insurance Database 2010 of the NHIRD as the data source, to examine the effectiveness of rehabilitation.

In terms of accuracy rate of all classifiers, we get the best effectiveness (78\%) while adopting the inpatient admission dataset and C4.5 to predict recurrence. We also find physical therapy, occupational therapy and speech therapy treatments during inpatient admission are the key factors to decrease the chance to recrudesce in the rehabilitation periods. The higher strength and frequency rehabilitation treatment is also the key influence variables in our high accuracy prediction model which means that is useful to lower the recurrence rate of stroke patients.
\end{abstract}

Key Words: Cerebral vascular accident, Stroke, Rehabilitation, Classified technology

\section{INTRODUCTION}

Stroke is a high incidence and high mortality of global diseases. Every year about thirty thousand early-onset stroke patients, of which about a million deaths, account for $7 \%$ of the population of deaths. On average, a person died of a stroke every 48 minutes. $^{[1]}$ Therefore, stroke is a serious health hazard to the people of the world's serious diseases.

There are one third of stroke survivors suffer from sequela that has long been in a disabling state in Taiwan these years.
Stroke patients with poor prognosis, not only affect their follow-up medical care costs, more severe impact (ex: restroke) on patients and their family quality of life. Many medical stroke-care guidelines afford proof that subsequent rehabilitation therapy on patients with functional rehabilitation plays an important role no matter Cerebral hemorrhage or Intracerebral Hemorrhage (ICH) or Ischemic Stroke patients. ${ }^{[2]}$ Rehabilitation therapy has been proven to be the only opportunities to achieve a good prognosis for disability

\footnotetext{
*Correspondence: Chia-Lun Lo; Email: allenlo.tw @gmail.com; Address: Department of Health-Business Administration, Fooyin University, 151 Jinxue Rd., Daliao Dist., Kaohsiung City 83102, Taiwan. 
with acute stroke feature of rehabilitation and decrease the chance of re-stroke. ${ }^{[3]}$ It is found that patients with stroke at this stage have a high potential for rehabilitation, rapid recovery of limb movements and function, and rejuvenation treatment is more effective in many clinical trials of patients with stroke ranging from three to six months after the onset of stroke. ${ }^{[4]}$ This period refers to "rapid restoration of function", commonly known as "Key rehabilitation period". Nevertheless, Kakuda et al. ${ }^{[5]}$ attempted to rehabilitate 204 patients with chronic stroke with a mean incidence of 5 years, using repetitive transcranial magnetic stimulation (rTMS) combined with high-frequency functional therapy. After 15 days of treatment found that upper limb movement quality and speed of execution activities are patient appears very significant improvement. Since the outcome of the treatment is not limited by age and number of years of the onset of the patient, so there is the golden period of rehabilitation or not, it is quite doubtful.

However, health insurance in Taiwan will limit the period from 6 to 12 months after a stroke to recovery payments due to the key rehabilitation period. Although the healthcare cost can be saved, but also caused many patients loss their treatment opportunities, especially those stroke more than one year. Therefore, it is difficult to determine the appropriate payment policy under the cost saving and the rights and interests of patients.

The existed researches on the rehabilitation of cerebral stroke have focused on the identification of different demographic characteristics among patients, the correlation between the past history and the prognosis. ${ }^{[6]}$ Only a few studies consider overall impact of the factor on the prognosis of patients. However, due to low viscosity of rehabilitation patients, the samples of existing studies generally in a single hospital, a single area, it is difficult to follow the actual follow-up of patients with hospital conditions caused by the results of bias. The National Health Insurance Research Database (NHIRD) in Taiwan, which covers nearly 23 million people, is representative of the health information. In addition, the development of the health care database underwriting sampling subsumed archives, long-term and continuous record contained all of the insured for medical treatment record since paid into, quite suitable for study of functional prognosis of patients tracking. As the impact of stroke patients with functional prognosis of many factors, and the various factors may affect each other, the impact of different degrees of prognosis. Conventional linear studies cannot handle complex multi-factor relationships, and cannot presuppose assumptions about unknown outcomes. Therefore, this study adopts machine learning techniques to construct the stroke recurrence prediction model, and use the characteristics of the
National Health Insurance Database, in order to truly reflect stroke patients' prognosis situations. The main purposes are as following:

(1) To summarize the factors influencing the prognosis of stroke patients and to find out the key factors.

(2) Verify stroke rehabilitation patient rehabilitation and relevance frequency and intensity in order to limit the empirical continue rehabilitation of stroke patients' appropriateness.

(3) Establishing a predictive model of stroke in stroke patients in order to use to assess patients' rehabilitation potential.

\section{Stroke disease and rehabilitation therapy}

Stroke refers to the brain blood flow obstruction, resulting in brain tissue hypoxia injury, resulting in a variety of cranial nerve defect symptoms. Stroke is a highly heterogeneous disease, although with different types, different brain injury caused by different clinical characteristics of the region, but often the patient caused a degree of disability. ${ }^{[7]}$ Stroke patients may produce various aspects of bodily function damage, such as the common side injury, hemiplegia, limb contracture; or the emergence of aphasia, dysarthria and other language barriers, causing great inconvenience to patients on their own lives.

According to different causes, clinical stroke can be divided into three types:

\section{(1) Ischemic stoke}

Ischemic stoke is the most common type of stroke, accounting for more than $80 \%$ of all stroke population. Vascular supply to the brain tissues of thrombus or other embolus blocked, causing asphyxia. Stroke can be subdivided into non-embolic ischemic stroke and embolic ischemic stroke.

(2) Cerebral hemorrhage or intracerebral hemorrhage ( $\mathrm{ICH})$

Cerebral hemorrhage or intracerebral hemorrhage caused by blood supply to the brain tissue blood vessels rupture, blood flow into the brain tissue, resulting in rapid increase in brain pressure, and squeeze the blood vessels near the brain tissue. When patient with high brain pressure easily lead to patients with dizziness, nausea, loss of consciousness or even death. The incidence of ICH is about $10 \%$.

(3) Transient ischemic stroke (TIC)

Transient ischemic commonly referred to as small stroke, short-term interruption of the brain blood flow, resulting in temporary partial neurological deficit, the symptoms usually disappear within 24 hours after the onset. 
In addition to the impact of self-care ability, the physical and psychological stress of patients and caregivers is also worthy of attention which is affecting the patient's physiology, psychology and behavior. ${ }^{[8]}$ Simon et al. ${ }^{[9]}$ showed a high correlation between the degree of caregiver depression and the degree of patient independence. The patients with stroke apoplexy state will result in patients, caregivers and society long-term and serious impact. ${ }^{[10]}$

Many scholars have different definitions of rehabilitation stages after stroke. 1) Acute phase: Refers to the stroke just occurred, usually contains 1 to 2 weeks of hospitalization. 2) Sub-acute phase: Stroke after about 2 weeks, continuing to 3 to 6 months. 3) Post-acute phase: Stroke occurs after 6 months, even for a few years. ${ }^{[11]}$ Patients, who are not as functional as those before treatment, require considerable active intervention in rehabilitation at any stage of recovery. ${ }^{[12]}$

The key period of rehabilitation is mainly due to some scholars believed that the human brain has the ability of "spontaneous neurological recovery". Although the current study of spontaneous recovery of the mechanism of spontaneous recovery is still not clear, but generally believed that spontaneous recovery occurred mostly in the brain injury of three to six months, some conservative scholars believe that the recovery within three months more obvious, the results of many clinical trial literatures are also consistent with these claims. ${ }^{[13]}$ Therefore, some scholars will be named as the "Plateau", patients at this stage recover faster, the recovery rate is more obvious, and the degree of improvement will then gradually decrease with the onset of the disease. ${ }^{[2,14]}$

However, some scholars have different results. Miltner et $a l .{ }^{[15]}$ for the treatment of cerebral stroke patients from 0.5 to 17 years of constraint-induced movement therapy (CIMT). The results showed that many patients have been in the chronic phase, after treatment, the affected limb function is still a significant improvement. Therefore, the concept of "healing effect only occurs in key rehabilitation period" is not absolutely confirmed. Many studies have shown that even patients who have progressed into the chronic stage of stroke have significantly improved treatment outcomes and improved functional outcomes through intensive stimulation, rehabilitation, and neuromuscular activation. ${ }^{[16]}$

The term key recovery period is easy to mislead patients to assess the effectiveness of rehabilitation treatment, and then produce a false expectation of the disease. Over the gold rehabilitation period of time, patients hold a negative attitude to rehabilitation therapy; even worse self-abandonment, lack of motivation, refused to accept rehabilitation therapy, resulting in psychological dependence, long-term loss can be seriously affected. Therefore, if the existing misconceptions be held, and the number of treatment be ignored, intensity of the impact of treatment effectiveness, long-term down, the patient's mentality, prognosis seriously affected, but also affected the entire family care burden and life quality, resulting in rehabilitation therapy ineffective.

In recent years, there are many studies to explore the functional outcome of stroke and mortality factors, but the results of the study there are many differences. Many previous studies have focused on demographic characteristics, and differences in past history have been used to explore the explanatory power of stroke progression. ${ }^{[4]}$ Although Lenze et al. ${ }^{[17]}$ explored the effect of rehabilitation on the prognosis of patients with stroke, the evidence is limited if only short-term functional prognosis is considered. On the other hand, because the clinical sample collection is not easy, many studies are only able to grasp long-term patients in the same hospital, once the patient to change the hospital, the accuracy of the study will be ignored it. In addition, many studies generally use functional independence scale to predict restroke. ${ }^{[18]}$ Although the scale used has high reliability and high efficiency, but the measured results can only represent a time point of the current functional performance of the patient. In fact, many studies see "patient re-stroke" as one of the poor prognosis. ${ }^{[19]}$ It can be seen that the recurrence of stroke is an important prognostic factor. ${ }^{[20]}$ In this study, first adopts machine learning techniques, which reveals the effect of rehabilitation therapy is an important factor in re-stroke. Our results can provide significant information in clinical decision support.

\section{METHOD}

\subsection{Databases}

This case-control study used data retrieved from the Longitudinal Health Insurance Database 2010 (LHID2010). The LHID2010, which is derived from medical claims records of the Taiwan National Health Insurance (NHI) program, includes original medical claims and registration files for 1,000,000 enrollees under the Taiwan NHI program. Taiwan National Health Institute randomly selected these 1,000,000 enrollees from all enrollees listed in the 2010 Registry of Beneficiaries ( $\mathrm{n}=23.72$ million). Prior studies and Taiwan National Health Institute have demonstrated the high validity of data derived from the NHI program. ${ }^{[21,22]}$

This study was exempt from full review by the Institutional Review Board of the Ditmanson Medical Foundation ChiaYi Christian Hospital because the LHID2010 consists of deidentified secondary data released to the public for research purposes. 


\subsection{Selection of cases and controls}

The patients in this study were selected from 1 January 2003 to 31 December 2007 in the outpatient clinic (including the outpatient department of hospitals and clinics) to receive the first diagnosis of stroke patients (ICD-9-CM Code 430.X438.X) $(n=4,286)$. We will receive the date of the first stroke diagnosis during this period as the index date. We include patients who have been diagnosed with stroke at least once by an accredited neurologist, but those with a stroke or only a single diagnosis (who concluded that they died or were wrong) $(n=3,105)$. We further excluded patients $(n=1,075)$ who had not undergone reconstructive therapy in outpatient data sheets and hospitalized medical records. In the final outcome, 466 medical records were included as the study medical records of the study (see Figure 1).

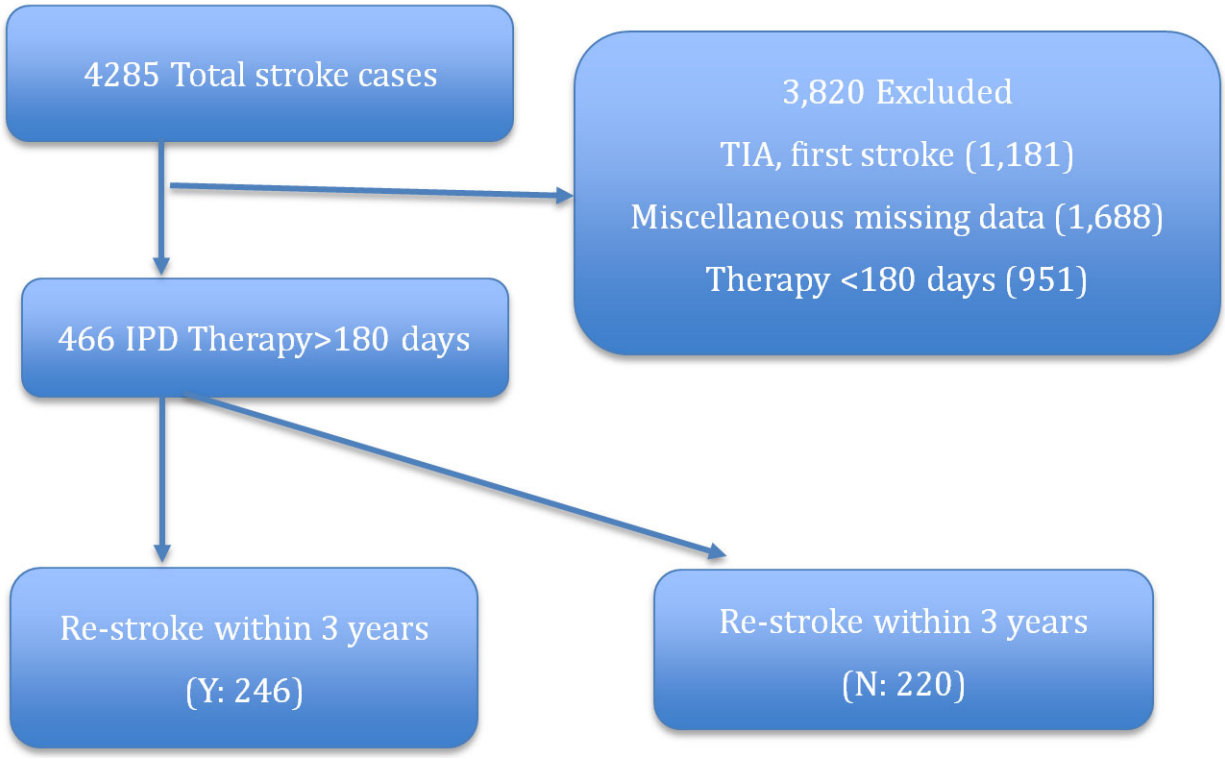

Figure 1. Flowchart showing derivation of the study sample

Corresponding to these patients, we also retrospectively reviewed the history of these 10 cases of illness occurring in these patients since the beginning of health insurance (1996) to the date of the last rehabilitative treatment performed by these patients as one of the independent variables from LHID2010. Also based on the literature, in addition to the type of stroke patients and hospital use of urinary tract as a recurrent factor of stroke, but also included in stroke when the age, sex and living area. Finally, the physiotherapy, functional therapy and language therapy were carried out in order to frequency, intensity and rehabilitation treatment of medical institutions as the level of its rehabilitation effects of factors. Dependent variable is used after three years of patients with stroke again because of admission and to determine the effectiveness of its gold treatment period. Twenty-two patients (49\%) had no further stroke after three years of treatment $(n=220)$.

\subsection{Exposure assessment}

The study was based on stroke cases in ICD-9-CM code 430438. Cases were diagnosed and treated at least once after leaving the hospital. We included only the first diagnosis of 4 stroke cases to achieve a balance between the starting points of the cases. In addition, the re-onset of the rehabilitation period is not spear considerations.

\subsection{Classification techniques}

To stratify risk groups for stroke rehabilitation patient, we performed a classification, including $\mathrm{C} 4.5,{ }^{[23]}$ regression tree (CART), and logistic regression (LGR).

Decision tree (DT) is a well-known and powerful supervised learning technique. ${ }^{[23]}$ It comprises a hierarchical structure comprising nodes and branches; and internal node represents an input variable, the branch of an internal node represents a subset of the values of the corresponding input variable, and a leaf node is associated with a value(or a class label)of the output variable. C4.5 and CART are the two most used DT-based learning techniques. They select their suitable input variable as an internal node and partition the training dataset into 2 subsets according to the Gain ratio and GINI index separately. LGR aims to predict the probability of the occurrence of an event by fitting data into a logistic function, thereby allowing inputs with any values to be transformed and confined to a value between 0 and 1. Each regression 
coefficient represents the corresponding variable's degree of contribution. A positive regression coefficient increased the probability of the output, while a negative value decreases the probability of the output.

We used Weka 3.6.1 open-source data mining software to perform all the analysis result. Specifically, all the module in weka was adopted its generate the module algorithms using a complete set of cohort data and variables. A 10-fold crossvalidation was used to assess the predictive ability of the tree model. To evaluate and to compare the performance of the classification models, we considered the following three metrics: prediction accuracy, sensitivity, and specificity.

Table 1. Statistics information for the collected clinical cases

\begin{tabular}{|c|c|}
\hline Variables & Statistics \\
\hline CVA & TIA: 486/Hemorrhagic stroke: 30 \\
\hline Catheter & Y: 188/N: 278 \\
\hline Age & $11 \sim 86 / \mu=62.305 / \sigma=12.51$ \\
\hline Sex & M: 288/F: 178 \\
\hline Area & $\begin{array}{l}\text { High urbanization: 134/Middle } \\
\text { Urbanization: 125/New town: 68/town: } 87 / \text { elderly } \\
\text { Town: 4/rural: } 26 / \text { remote village: } 22\end{array}$ \\
\hline Hypertension & Y: 347/N: 119 \\
\hline Diabetes & Y: 172/N: 294 \\
\hline MI & $\mathrm{Y}: 12 / \mathrm{N}: 454$ \\
\hline Atrial Fibrillation & $\mathrm{Y}: 18 / \mathrm{N}: 448$ \\
\hline $\mathrm{HF}$ & $\mathrm{Y}: 50 / \mathrm{N}: 416$ \\
\hline COPD & Y: 139/N: 327 \\
\hline Dementia & $\mathrm{Y}: 9 / \mathrm{N}: 457$ \\
\hline Depression(Premorbid, after illness) & $\begin{array}{l}Y: 6 / N: 460 \\
Y: 26 / N: 440\end{array}$ \\
\hline TIA history & $\mathrm{Y}: 23 / \mathrm{N}: 443$ \\
\hline Family history & Y: 112/N: 344 \\
\hline Hospital level & $\begin{array}{l}\text { Medical center: } 104 \\
\text { Regional hospital: } 135 \\
\text { Community hospital: } 36 \\
\text { Rehabilitation clinical: } 191\end{array}$ \\
\hline Rehabilitation therapy high frequency & $\begin{array}{l}\text { OT:Y: } 207 / \mathrm{N}: 259 \\
\text { PT:Y: 292/N: } 174 \\
\text { ST:Y: } 31 / \mathrm{N}: 435\end{array}$ \\
\hline Rehabilitation therapy high intensity & $\begin{array}{l}\text { OT:Y: 243/N: } 223 \\
\text { PT:Y: } 318 / \mathrm{N}: 148 \\
\text { ST:Y: } 87 / \mathrm{N}: 379\end{array}$ \\
\hline Re-stroke within 3 years & $\mathrm{Y}: 246 / \mathrm{N}: 220$ \\
\hline
\end{tabular}

\section{RESULTS}

A total of 4,285 stroke diagnosis patients were identified and 3,839 stroke patients were excluded from our study. Of the 446 stroke patients, 246 patients $(55 \%)$ were re-stroke within Published by Sciedu Press
3 years that the "Y" group slightly more than "N" group. The distribution of diagnostic categories and therapy characteristics also varied between the 2 groups. The variables and its statistics information are as shown in the Table 1. 
Table 2. Correlation between different therapies and re-stroke $(\mathrm{N}=446)$

\begin{tabular}{llll}
\hline Variables & Beta & Variables & Beta \\
\hline OPD OT intensity & Beta $=0.271$ & IPD OT intensity & Beta $=-0.630^{*}$ \\
OPD PT intensity & Beta $=-0.193$ & IPD PT intensity & Beta $=-1.255^{* * *}$ \\
OPD ST intensity & Beta $=-0.374$ & IPD ST intensity & Beta $=-0.104$ \\
OPD OT frequency & Beta $=-0.219$ & IPD OT frequency & Beta $=0.343$ \\
OPD PT frequency & Beta $=-0.073$ & IPD PT frequency & Beta $=-0.657^{* *}$ \\
OPD ST frequency & Beta $=0.875$ & IPD ST frequency & Beta $=-0.469$ \\
\hline
\end{tabular}

Note. OT: occupational therapy; PT: physical therapy; ST: speech therapy; OPD: outpatient department; IPD: inpatient department.

Table 2 presents the correlation among intensity and frequency of the different therapies and re-stroke. We found that intensity of occupational therapy, physical therapy, and frequency of physical therapy are significantly correlation with concurrence of re-stroke. Several therapies are nega- tively correlated to the re-stroke, but there is no significant statistical relationship with re-stroke. Therefore, the quality of physical therapy care were verified the most important factor to the stroke patients.

Table 3. Performance evaluation of the classifiers

\begin{tabular}{llll}
\hline Classifier & Accuracy & Sensitivity & Specificity \\
\hline C4.5 & 0.78 & 0.81 & 0.74 \\
CART & 0.74 & 0.76 & 0.71 \\
LGR & 0.68 & 0.468 & 0.89 \\
\hline
\end{tabular}

Table 4. Ranking of variables for the re-stroke groups

\begin{tabular}{lll}
\hline Attribute & Values & Rank \\
\hline IPD PT intensity & 0.108742 & 1 \\
IPD PT frequency & 0.072435 & 2 \\
IPD OT intensity & 0.0676344 & 3 \\
IPD OT frequency & 0.0375442 & 4 \\
IPD ST intensity & 0.0224513 & 5 \\
Living area & 0.0138273 & 6 \\
IPD ST frequency & 0.0118785 & 7 \\
IPD PT intensity & 0.0060437 & 8 \\
CPOD & 0.0047563 & 9 \\
IPD ST intensity & 0.003749 & 10 \\
Catheter & 0.0033372 & 11 \\
Hypertension & 0.0032564 & 12 \\
TIF history & 0.0023658 & 13 \\
Atrial fibrillation & 0.0022529 & 14 \\
CVA type & 0.0022465 & 15 \\
HL & 0.0002365 & 25 \\
Depression (after) & 0.0001329 & 26 \\
Dementia & 0.0000437 & 27 \\
Depression (premorbid) & 0.0000293 & 28 \\
\hline & & \\
\hline
\end{tabular}

The evaluation results summarize the evaluation results for complete clinical cases in Table 3. The accuracy of C4.5, CART, and LGR are 0.78, 0.74, and 0.68. Of the studied characteristics, the $\mathrm{C} 4.5$ method identified re-stroke patient as the best single discriminator between the patients with re-stroke chance and without the restrike chance. The results showed that the correct rate was $78 \%$ in the model of prognosis established by the rehabilitation frequency and intensity of hospitalization. In general speaking, the correct rate of model prediction of outpatient rehabilitation therapy was just $52.69 \%$. The results of this study are in line with previous findings in the literature, ${ }^{[24,25]}$ which emphasizes the fact that "intensive treatment during hospitalization has a positive impact on patient outcomes". The results of this study suggest that clinicians should be able to provide sufficient treatment intensity and treatment frequency for stroke patients during hospitalization. In addition to having to cope with changes in physical condition at the onset of a stroke, it is important to establish awareness of the disease and the safety of the surrounding environment. ${ }^{[24]}$ To the physician point, the chance of the re-stroke cannot determination clearly before it happen, therefore, the results in this study are also shown that regardless of what type of classifier we used, the accuracy is significantly higher than the guess by the physician guess. Therefore, establishing a predictive model of stroke in stroke patients in order to use to assess patients' rehabilitation po- 
tential by machine learning can provides evidence to decide the benefits to keep rehabilitation therapy.

For finding out the key factors influencing the prognosis of stroke patients, we also calculated the score of each input variable in this study. Based on the gain ratio of each input variable associated with the dependent variable in Weka, we ranked all input variables and list top 15 factors shown in Table 4. As shown, rehabilitation therapy of PT, OT in IPD is the critical variables affecting prediction accuracy for the re-stroke groups.

The professional therapist in the hospital during the training of patients in addition to limb movements, but also the need for patients and caregivers for health education: including awareness of the disease, induced factor control, patient translocation and movement skills, home security considerations, related aids assessment and recommendations. It is very important for the stroke patients and their families to establish the right disease awareness and strengthen the follow-up care skills, which can effectively reduce the recurrence rate of cerebral stroke and achieve good prognosis. ${ }^{[26]}$ Therefore, providing high-intensity, high-frequency rehabilitation therapy during hospitalization is beneficial for improving prognosis. ${ }^{[25]}$ At the same time, consider other related prognostic factors such as the control of co-morbidities, the distribution of medical resources in the patient's living areas, medical staff in addition to providing stroke patients with limb movement training or daily life function training, but also need to provide immediate psychological support, ${ }^{[27,28]}$ and to educate patients about the concept of good health and to strengthen the patient's self-health management skills, ${ }^{[29,30]}$ reducing the rate of recurrent stroke.

On the other hand, although the effect of rehabilitative therapy in outpatient care is less significant for prognosis, maintaining functional status is an important therapeutic goal for patients with chronic stroke. Continuing to implement appropriate exercise programs and maintaining physical activity is a self-management program that is highly emphasized by many investigators for chronic patients. ${ }^{[29,31]}$ Thus, even when patients are discharged post-disability of varying degrees, it should continue to receive outpatient rehabilitation therapy. Clinically, many patients with stroke due to pain, emotional or mobility inconvenience and other factors bedridden, resulting in rapid decline in heart and lung function, and even the implementation of simple daily activities, to maintain the sitting position is also quite laborious. Long-term inactivity coupled with chronic disability, aging effects, coupled with poor medical compliance, not properly control the associated risk factors, leading to deteriorating physical function, virtually greatly increased the risk of re-stroke. ${ }^{[29]}$

Published by Sciedu Press
Many chronic stroke patients, because consciousness needs to continue to seek outpatient rehabilitation therapy; helpless physician health care is limited to nuclear puncturing mechanism for the implementation of the stroke patients, often due to health care should be standardized to reduce the intensity of the rehabilitation treatment of such patients, resulting in patient. The unit time for outpatient rehabilitation has been severely curtailed. How to meet the needs of patients and the cost of medical expenses to achieve a balance between the two is a very important and urgent topic. Through our clinical judgment, we can grasp the chance of recovery of stroke patients, maintenance of rehabilitation therapy, and adjust the intensity and frequency of treatment, in order to reduce the recurrence.

\section{Discussion AND CONCLUSION}

Stroke is a complex and heterogeneous disease, ${ }^{[30]}$ but with the development of medical knowledge, technology can be combined with traditional rehabilitation therapy to achieve better therapeutic outcomes. For example, many recent studies have combined high and low frequency craniocerebral magnetic stimulation and conventional localized induction therapy, ${ }^{[5]}$ as well as the use of visual-motor feedback technology such as Wii, XBOX-Kinect, ${ }^{[32]}$ or the recent rise of mirror therapy ${ }^{[33]}$ The effectiveness of these emerging therapies have been clinically acclaimed and a growing number of investigators have attempted to apply these findings extensively to patients with stroke at different ages and stages, with many traditional therapeutic limitations reaching a breakthrough development. According to the study by, ${ }^{[30]}$ many stroke patients are most worried about, and fear is the recurrence of stroke. The results of this study are based on the fact that stroke patients with stable physiological home signs face an unknown end time rehabilitation treatment of good clinical assistance tools.

Most of the results show that improved treatment intervention, its effectiveness is no longer confined to a specific stage of stroke patients, but for most patients with stroke. However, because the length of rehabilitation therapy still depends on the patient's recovery status may be, in order to save health insurance costs, there is a key rehabilitation period to save insurance costs.

In this study, in addition to confirming the importance of the gold rehabilitation period, we also try to establish a prognosis model of stroke patients through supervised learning technology, and explore the possible factors of recurrent stroke patients. Our results confirm that the strength and frequency of rehabilitative therapy are important factors influencing the patients' re-stroke. This conclusion is consistent with the results of many previous studies. The intensity of rehabili- 
tation therapy, on behalf of the patient each time to receive rehabilitation treatment unit length of time, which means that the stronger the intensity of treatment units in patients with longer. According to the ${ }^{[34]}$ study, stroke patients during hospitalization, rehabilitation treatment time of about 30 to 60 minutes per day, but with the longer the time of illness, daily rehabilitation time and frequency are gradually reduced. Many patients even only performed for 6 months to stop treatment, rehabilitation needs can not be met.

With the increasingly stringent review of health insurance, the benefits of treatment for patients were sacrificed, the loss of recovery of valuable opportunities. From the results of this study, we can see that the PT and OT received during hospitalization are more related to the recovery after the hospitalization. Therefore, this study suggests that patients should be rehabilitated during the hospitalization period, and then rehabilitated with the overall time limit. Declaring as an enabling force allows healthcare providers to increase the motivation for providing "effective" treatment, which is a win-win future for patients or health-care providers.

Since this study sources of secondary data research, failed to accurately grasp the extent of physical and mental energy lost after the stroke patients disease. Future studies suggest that patients with stroke can extend the tracking time, and for the patient's disability status of the detailed record and track in detail, do master the patient's functional recovery. Second, because this study only on the intensity and frequency of rehabilitation therapy to study, if the treatment of various items (such as the functional treatment of daily life training, coordination of training) for analysis, it should be able to better grasp the rehabilitation of patients' long-term prognosis, to provide clinical treatment as a reference direction for treatment to help stroke patients get a good functional prognosis.

\section{REFERENCES}

[1] Ministry of Health cand Welfare. Health Statistics in Taiwan. Retrieved from http://www.mohw.gov.tw/cht/DOS/Statistic. aspx?f_list_no $=312 \&$ fod_list_no $=2747$

[2] Langhorne P, Bernhardt J, Kwakkel G. Stroke rehabilitation. The Lancet. 2011; 377(9778): 1693-702. https ://doi.org/10.101 6/s0140-6736(11)60325-5

[3] Murie-Fernández M, Irimia, Martínez-Vila E, et al. Neurorehabilitation after stroke. Neurología. 2010; 25(3): 189-96. https : //doi.org/10.1016/S0213-4853(10)70008-6

[4] Vahlberg B, Cederholm T, Lindmark B, et al. Factors Related to Performance-Based Mobility and Self-reported Physical Activity in Individuals 1-3 Years after Stroke: A cross-sectional Cohort Study. Journal of Stroke and Cerebrovascular Diseases. 2013; 22(8): 426-34. PMid:23721615. https://doi.org/10.1016/j.jstrokecereb rovasdis. 2013.04 .028

[5] Kakuda W, Abo M, Shimizu M, et al. A multi-center study on lowfrequency rTMS combined with intensive occupational therapy for upper limb hemiparesis in post-stroke patients. Journal of NeuroEngineering and Rehabilitation. 2012; 9(4): 1-11.

[6] Naess H, Lunde L, Brogger J, et al. Depression predicts unfavourable functional outcome and higher mortality in stroke patients: The Bergen Stroke Study. Acta Neurologica Scandnavica. 2010; 190: 34 8. PMid:20586733. https ://doi.org/10.1111/j.1600-0404 2010.01373. $\mathrm{x}$

[7] Hung MC, Hsieh CL, Hwang JS, et al. Estimation of the long-term care needs of stroke patients by integrating functional disability and survival. PLOS ONE. 2013; 8(10): 75605. PMid:24124500. https://doi.org/10.1371/journal.pone.0075605

[8] Rachpukdee S, Howteerakul N, Suwannapong N, et al. Quality of Life of Stroke Survivors: A 3-Month Follow-up Study. Journal of Stroke and Cerebrovasc Diseases. 2013; 22(7): 70-8. PMid:22749628. https://doi.org/10.1016/j.jstrokecereb rovasdis.2012.05.005

[9] Simon C, Kumar S, Kendrick T. Formal support of stroke survivors and their informal carers in the community: a cohort study. Health \&
Social Care in the Community. 2008; 16(6): 582-92. PMid:18371168. https ://doi.org/10.1111/j.1365-2524.2008.00782.x

[10] Qiu Y, Li S. Stroke: Coping strategies and depression among Chinese caregivers of survivors during hospitalisation. Journal of Clinical Nursing. 2008; 17(12): 1563-73. https://doi.org/10.1111/j . 1365-2702.2007.02156.x

[11] Murie-Fernández M, Ortega-Cubero S, Carmona-Abellán M, et al. Time is brain: Only in the acute phase of stroke? Neurología. 2012; 27(4): 197-201. PMid:21890240. https://doi.org/10.1016/j . $\mathrm{nrl.2011.06.007}$

[12] Duncan PW, Zorowitz R, Bates B, et al. Management of adult stroke rehabilitation care: a clinical practice guideline. Stroke. $2005 ; 36(9)$ : 100-43. PMid:16120836. https://doi.org/10.1161/01.STR.0 $000180861.54180 . \mathrm{FF}$

[13] Kwakkel G, Kollen B, Lindeman E. Understanding the pattern of functional recovery after stroke: facts and theories. Restorative Neurology and Neuroscience. 2004; 22(3-5): 281-99. PMid:15502272.

[14] Verheyden G, Nieuwboer A, De Wit L, et al. Time course of trunk, arm, leg, and functional recovery after ischemic stroke. Neurorehabil Neural Repair. 2008; 22(2): 173-9. PMid:17876069. https : //doi.org/10.1177/1545968307305456

[15] Miltner WH, Bauder H, Sommer M, et al. Effects of constraintinduced movement therapy on patients with chronic motor deficits after stroke: A replication. Stroke. 1999; 30(3): 586-92. PMid:10066856. https ://doi.org/10.1161/01.STR.30.3.58 6

[16] Teasell R, Meyer MJ, McClure A, et al. Stroke rehabilitation: An international perspective. Topics in Stroke Rehabilitation. 2009; 16(1): 44-56. PMid:19443347. https ://doi .org/10.1310/tsr1601-4 4

[17] Lenze EJ, Munin MC, Quear T, et al. Significance of poor patient participation in physical and occupational therapy for functional outcome and length of stay. Archives of Physical Medicine and Rehabilitation. 2014; 85(10): 1599-601. https://doi.org/10.101 6/j.apmr.2004.03.027

[18] Ones K, Yilmaz E, Cetinkaya B, et al. Quality of life for patients poststroke and the factors affecting it. Journal of Stroke Cerebrovasc 
Diseases. 2005; 14(6): 261-6. PMid:17904035. https://doi.or $\mathrm{g} / 10.1016 / \mathrm{j} \cdot \mathrm{j}$ strokecerebrovasdis .2005 .07 .003

[19] Fatahzadeh M, Glick M. Stroke: Epidemiology, classification, risk factors, complications, diagnosis, prevention, and medical and dental management. Oral Surgery, Oral Medicine, Oral Pathology, Oral Radiology, and Endodontology. 2006; 102(2): 180-91. PMid:16876060. https://doi.org/10.1016/j.tripleo.2005.07.031

[20] Appelros P, Nydevik I, Viitanen M. Poor outcome after first-ever stroke: predictors for death, dependency, and recurrent stroke within the first year. Stroke. 2002; 34(1): 122-6. https://doi.org/10.1 161/01.STR.0000047852.05842.3C

[21] Chiu YL, Kao S, Lin HC, et al. Benign prostatic enlargement is not associated with diabetes: A population-based study. Andrology. 2015; 3: 933-6. PMid:26216209. https ://doi .org/10.1111/an dr. 12069

[22] Chen YC, Yeh HY, Wu JC, et al. Taiwan's national health insurance research database: administrative health care database as study object in bibliometrics. Scientometrics. 2011; 86: 365-80. https://doi.org/10.1007/s11192-010-0289-2

[23] Quinlan JR. C4. 5: Programs for machine learning. San Francisco, CA, US: Morgan kaufmann; 1993.

[24] Jette DU, Latham NK, Smout RJ, et al. Physical therapy interventions for patients with stroke in inpatient rehabilitation facilities. Physical Therapy. 2005; 85(3): 238. PMid:15733048.

[25] Karges J, Smallfied S. A description of the outcomes, frequency, duration, and intensity of occupational, physical, and speech therapy in inpatient stroke rehabilitation. Journal of Allied Health. 2009; 38(1): $1-10$.

[26] Croquelois A, Bogousslavsky J. Risk awareness and knowledge of patients with stroke: results of a questionnaire survey 3 months after stroke. Journal of Neurology, Neurosurgery \& Psychiatry. 2006; 77(6): 726-8. PMid:16549417. https://doi.org/10.1136/jn np. 2005. 078618

[27] Koenig KL, Whyte EM, Munin MC, et al. Stroke-related knowledge and health behaviors among poststroke patients in inpatient rehabilitation. Archives of Physical Medicine and Rehabilitation. 2007; 88(9): 1214-6. PMid:17826471. https ://doi .org/10.1016/j . apmr. 2 007.05 .024
[28] Sullivan KA, White KM, Young RM, et al. Developing a stroke intervention program: What do people at risk of stroke want? Patient Education and Counseling. 2008; 70(1): 126-34. PMid:17997262. https://doi.org/10.1016/j.pec.2007.09.006

[29] Sacco RL, Adams R, Albers G, et al. Guidelines for prevention of stroke in patients with ischemic stroke or transient ischemic attack: A statement for healthcare professionals from the American Heart Association/American Stroke Association Council on Stroke: co-sponsored by the council on cardiovascular radiology and intervention: the American Academy of Neurology affirms the value of this guideline. Circulation. 2006; 113(10): e409-49. PMid:16534023.

[30] Townend E, Tinson D, Kwan J, et al. Fear of recurrence and beliefs about preventing recurrence in persons who have suffered a stroke. Journal of Psychosomatic Research. 2006; 61(6): 747-55. PMid:17141662. https://doi.org/10.1016/j.jpsychores.2 006.09 .006

[31] Gordon NF, Gulanick M, Costa F, et al. Physical activity and exercise recommendations for stroke survivors an American heart association scientific statement from the council on clinical cardiology, subcommittee on exercise, cardiac rehabilitation, and prevention; the council on cardiovascular nursing; the council on nutrition, physical activity, and metabolism; and the stroke council. Circulation. 2004; 109(16): 2031-41. PMid:15117863. https : //doi.org/10.1161/01.CIR.0000126280.65777.A4

[32] Lee G. Effects of Training Using Video Games on the Muscle Strength, Muscle Tone, and Activities of Daily Living of Chronic Stroke Patients. Journal of Physical Therapy Science. 2013; 25(5): 595. PMid:24259810. https://doi.org/10.1589/jpts. 25.59 5

[33] Michielsen ME, Selles RW, van der Geest JN, et al. Motor Recovery and Cortical Reorganization After Mirror Therapy in Chronic Stroke Patients A Phase II Randomized Controlled Trial. Neurorehabilitation and Neural Repair. 2011; 25(3): 223-33. PMid:21051765. https://doi.org/10.1177/1545968310385127

[34] Schaechter JD. Motor rehabilitation and brain plasticity after hemiparetic stroke. Progress in Neurobiology. 2004; 73(1): 61-72. PMid:15193779. https://doi.org/10.1016/j.pneurobio.20 04.04 .001 